\title{
EARLY ADMINISTRATION OF ERYTHROPOIETIN RINCREASE THE RISK OF RETINOPATHY OF PREMATURITY
}

\author{
M.G. Terrin ${ }^{1}$, A. Faiella ${ }^{2}$, A. Magli ${ }^{1}$, F. Messina ${ }^{2}$, G. Terrin $^{3}$ \\ ${ }^{I}$ Department of Ophtalmology, University of Naples 'Federico II', ${ }^{2}$ Division of Neonatology, Evangelic \\ Hospital "V. Betania", Naples, ${ }^{3}$ Department of Women Health, University of Rome 'La Sapienza', Rome, \\ Italy
}

Background and aims: An association between the use of erythropoietin (EPO) and retinopathy (ROP) in very low birth weight (VLBW) newborns has been suggested. It has not been clearly defined if this risk depend by the time of administration (early or late) or by the dosage of recombinant EPO.

Methods: Using data prospectively collected by VLBW newborns consecutively observed in Neonatal Intensive Care Units (NICU) during two years, we compared two modalities of EPO administration: early (EPO initiated at $<8$ days of life) or late (EPO initiated $>14$ day of life only in the case of hematocrit $<34 \%$ without symptoms of anaemia), at $900 \mathrm{U} / \mathrm{kg} /$ week given subcutaneously in three divided doses until the age of 9 weeks. Outcomes were the rate of subjects with ROP of any stage and severe forms requiring cryotherapy or laser photocoagulation.

Results: We enrolled 98 newborns (birth weight $1080 \pm 271 \mathrm{~g}$; gestational age $28 \pm 3$, male 50 ): 40 in the early group, and 58 in the late group. Study group were comparable for the main clinical characteristics. The occurrence of ROP any stage resulted increased in a group of newborns receiving EPO early (17/40) compared with those treated with EPO later in the life $(7 / 58, \mathrm{p}<0.001)$. Severe forms of ROP not differs among groups (1 per group). Early administration of EPO increase the risk of ROP any stage (OR 2.4; $95 \%$ CI $1.2-4.5)$.

Conclusions: Early administration of recombinant EPO increase the risk of retinopathy of prematurity. 